\title{
Research on the Key Techniques of Augmented Reality Navigation
}

\author{
Rui.gu \\ Information Engineering \\ Suzhou Industrial Park Institute of Services Outsourcing \\ Suzhou, China \\ gur@siso.edu.cn
}

\author{
Jiale.gu \\ Teaching service center \\ Suzhou Industrial Park Institute of Services Outsourcing \\ Suzhou, China \\ gujl@siso.edu.cn
}

\begin{abstract}
With the rapidly development of location-based services, Using augmented reality technology to develop LBS system can greatly improve human computer interaction. In the article, we design an augmented reality system based LBS on android device, mainly discussed the geodetic coordinate transformation, openGL coordinates of the virtual data to the mobile phone interface mapping algorithm, which has an important guiding significance for the development of similar system.
\end{abstract}

Keywords—augmented reality; LBS; Geodetic coordinate system

\section{INTRODUCTION}

Augmented reality(AR) is the use of computer graphics and visualization technology to generate virtual objects virtual objects accurately "placed" in the real environment to achieve the virtual graphics and real environment, which achieves the virtual graphics and real environment integration, thereby enhancing the user's visual experience.

With the rapid development of smart phone technology, most of the smart phone has a powerful CPU, cameras, sensor, GPS and compass functions, which have fully meet the augmented reality of the hardware requirements[1]. Meanwhile the speed of mobile Internet could to meet the needs of network queries and data transmission, With a small, portable, Convenient, high penetration rate of handheld devices has become the ideal platform to enhance the reality of technology. therefore, research on AR systems on handheld devices has become an urgent need.

\section{AugMeNTED REALITY APPLICATION MODE}

\section{A. Card - based application model}

The main representative of the open source project ARToolKit, which is mainly to save the image Maker into local, through image recognition technology to identify the current image in the Maker[2]. And then overlays the relevant information on the Maker, which must install the camera, display, and Maker images. The main technologies are threedimensional registration algorithm, spatial visual matching algorithm and so on. Qualcomm has released an augmented reality sdk development kit for the Android platform to enable developers to develop such card-based augmented reality

Sponsor: Jiangsu Province Higher Vocational Colleges and universities domestic senior visiting scholar project applications.

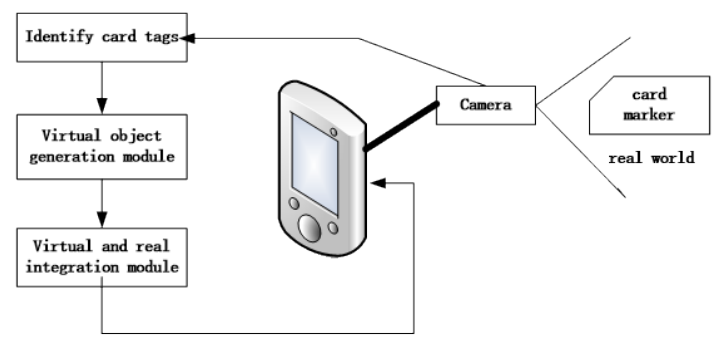

Fig. 1. Card Recognition Augmented Reality

\section{B. GPS+Sensor based application model}

The application model mainly through the GPS to obtain longitude, latitude and altitude, the geomagnetic Sensor (electronic compass) to obtain the direction of orientation, the acceleration Sensor to obtain the angle of tilt[3]. And then obtain the relevant information based on these location information. According to the information superimposed display. It must install the camera, display, GPS, geomagnetic GPS, acceleration sensor. The main technologies are virtual data to the mobile phone interface mapping algorithm, geodetic coordinate system and openGL ES 3D coordinate system conversion algorithm. Wikitude drive and Wikitude World Browser is based on this technology Developed by wikitude. The World Browser and layar almost the same principle, it can be a number of different types of information colleagues superimposed on the camera, Wikitude drive is a combination of factual traffic information. And webcam information for augmented reality navigation software.

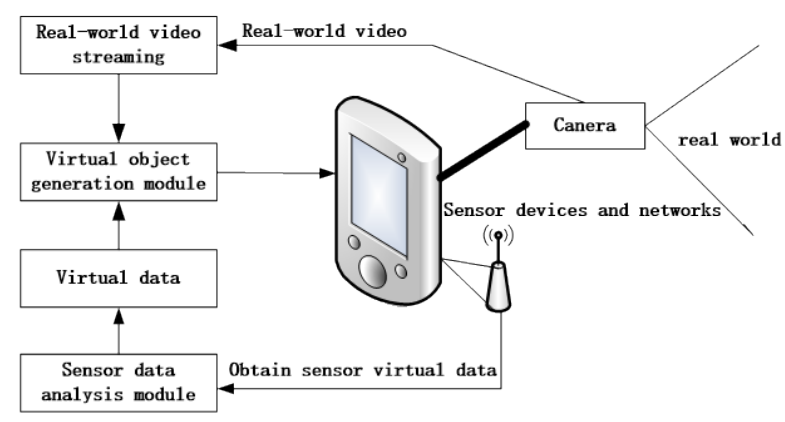

Fig. 2. GPS+Sensor based application model 


\section{THE PRINCIPLE OF GPS+SENSOR BASED APPLICATION MODE}

GPS+Sensor based application mode, which firstly gets the current location of the user and latitude ,longitude and height by GPS sensor, and then use latitude and longitude information as a parameter, call the map service map data service, which access to the data within a certain range around the current, the data also contains latitude and longitude information. Secondly, it obtains the direction information of the sensor on the android electronic compass. According to the current direction of the mobile phone, the data from the downloaded data is filtered according to the current data. The acceleration sensor converts the coordinates of the geodetic coordinate system into 3D coordinates on the openGL ES. Finally, in the openGL 3D coordinate system draw nearby data and the camera's video stream overlay, to achieve the purpose of combining virtual data and video streams。

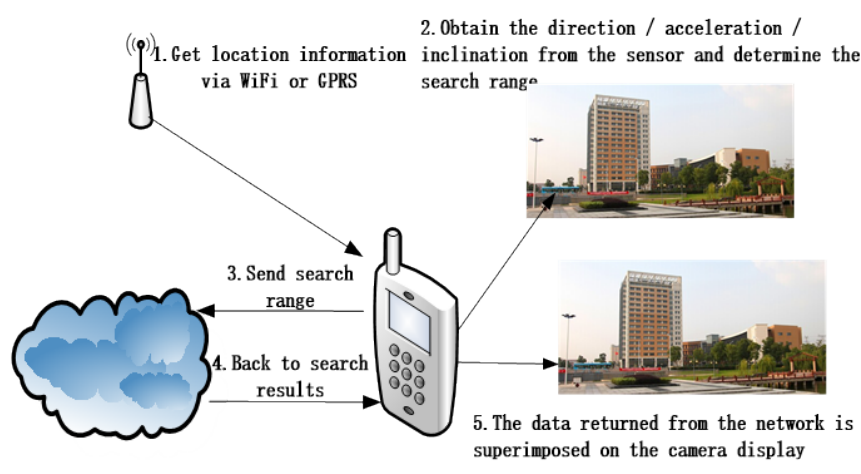

Fig. 3. The principle of GPS+Sensor based application model

If the location information is not accurate, it will lead to the information obtained from the internet and the user's current location information are not accurate, leading to data on the handheld device error. Similarly, if the information from the internet to get the GPS is not correct, the same will lead to the error displayed by the handheld terminal.

\section{AnAlysis of Key TeChNOlOgies of Augmented REALITY BASED ON LBS}

\section{A. Geodetic coordinate system and openGL ES 3D coordinate transformation algorithm}

In the android platform, we the need to use its 3D coordinate system in case using openGL ES drawing objects. Consider the state of the handheld device changes, you can directly draw[6]. If you do not consider handheld device status changes, you can directly draw. However, in order to simulate objects on the 3D coordinate system (gesture, deflection, tilt) on the handheld, it is necessary to use the sensor data. In android, acceleration sensors and gravity sensors can change the data to simulate the action on the handheld device, we need to change these sensor data matrix mapped to the openGL ES 3D coordinate system, and finally to draw objects on OpenGL 3D could simulate the effect of a variety of handheld devices.
As the sensor data changes, an array of sensor changes can be obtained in the register method, accels[0],accels[1],accels[2] the acceleration in the $\mathrm{x}, \mathrm{y}, \mathrm{z}$ axis value respectively. Then call getRotationMatrix (float 口 R, float 口 I, float[] gravity, float[] geomagnetic), the function of the method is coordinate the system equipment into the world coordinate system, and the results are converted into tilt matrix I and the rotation matrix $R$. Specific implementation process is as follows:

(1) Define R as the identity matrix, when the device is aligned with the world coordinate system, that is the device's $\mathrm{X}$-axis points east and the $\mathrm{Y}$-axis points to the north pole and the device is facing the sky.

(2) Defining I matrix to transform a geomagnetic vector into a rotation matrix of gravity in the same world coordinate space, the I-matrix is simply a matrix rotating around $\mathrm{X}$.

(3) According to the acceleration sensor and the geomagnetic sensor obtained after the rotation matrix need to continue to transform the matrix, because the resulting rotation matrix relative to the earth coordinate system, also need to be converted into openGL ES in the 3D coordinate system corresponding matrix.

Android provides remapCoordinateSystem (float [] inR, int $\mathrm{X}$, int $\mathrm{Y}$, float [] outR), which can rotate the matrix in accordance with the three directions of the system equipment mapping to a specified coordinate system.

\section{B. Virtual data to mobile phone interface mapping}

The virtual data is finally drawn and rendered by the openGL ES 3D and displayed on the handset display, but the display size of the phone is limited and does not display all the data[4][5]. It is necessary to filter the surrounding objects in the near and far directions and display the appropriate data accurately. In addition to the need to ensure that their own near the data show the greater the farther the object shows the farther away, so we need to find the right way.

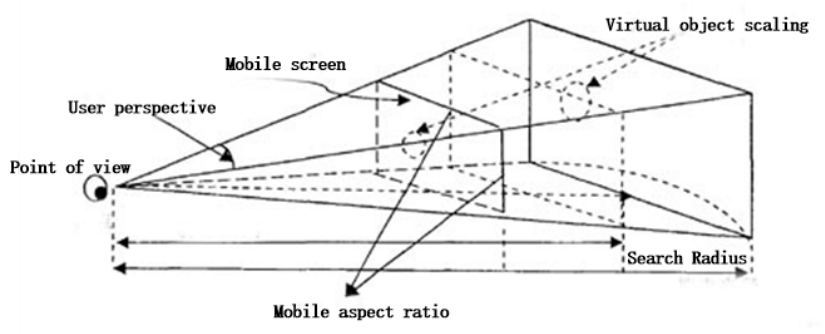

Fig. 4. Virtual data to mobile phone interface mapping

As can be seen from the Fig., because the virtual data can be obtained with the current distance, the phone's aspect ratio, the user's perspective and other information, so the most distant objects can be mapped through the viewport to the mobile phone screen, the other virtual data such as Objects within the distance box can also be mapped to the phone screen, and according to the distance can be calculated object scaling, and the user perspective data will automatically turn with the phone to meet the requirements of the data is accurately mapped to the phone. Specific implementation process is as follows: 
(1) Calculate the distance between the virtual data and the current position, relative to the $\mathrm{x}, \mathrm{y}$ values of the geodetic coordinate system.

(2) The data is mapped from the openGL 3D coordinate system to the phone window coordinate system.

(3) After the position of the virtual object is determined, the virtual object is rotated, enlarged, and reduced. Rotation here is when the virtual object and the current location of the direction is determined after the mobile phone at this time, you need to rotate the virtual data of a certain angle.

Through the calculation and transformation of the virtual data, it can be accurately mapped to the openGL 3D coordinate system, and can accurately simulate the true state of the data[7][8].

\section{CONCLUSION}

Augmented reality is the real world in which people are exposed to virtual electronic information to enhance realworld information, which can combine virtual world and realworld to give users a whole new experience. LBS-based augmented reality is a kind of use of smart phones on the GPS to obtain the user's current data, and through the smart phone sensor to virtual data will be displayed on the camera, to achieve the purpose of combining virtual and realistic, to provide users with Convenient and efficient data services.

\section{REFERENCES}

[1] Wagner D, Schmalstied G D. ARToolKit on the pocketPC platform SecondIEEE Intl Augmented Reality ToolKit Workshop. Tokyo, Japan:IEEE, 2003, 28:34

[2] Chen W C, Xiong Y Gao J. Efficient extraction of robust image features on mobile devices Int Symp on Mixed and Augmented Reality(ISMAR,07). NewYork USA: ACM, 2007, 20-27

[3] Dieter Schmalatieg. Anton Fuhrmann. Gerd Hesina. Zsolt Szalavari LMiguel Encarna Michael Gervautz and Werner Purgnthofer The studierstube augmented reality project, 2002, (1): 30-40

[4] Feiner S,Maelntyre B,Seligmann D.Knowledge-based augmented reality[J].Communications of the ACM,1993,36(7),52 62.

[5] Chunyong Ma, Yongyang Qi, Yong Chen, Yong Han, Ge Chen. VRGIS: An Integrated Platform of VR Navigation and Gis Analysis for City/Region Simulation[R]. VRCAI2008, Association for Computing Machinery,Inc, Singapore, 2008-12-08.

[6] Ionitescu S. On Using Augmented Reality Technologies to Improve the Interaction between Realand Virtual Spaces[J].The 7th International Conference on Virtual Learning ICVL 2012.

[7] Tse,Hiu-yeung. Virtual Campus Navigation of Android Smart Phone Application. Run RunAllison, R. S., Eizenman, M., \& Cheung, B. S. K. Combined head and eye tracking system for dynamic testing of the vestibular system. IEEE Transactions on Biomedical Engineering, 1996, 43:1073-1082

[8] R.Y.Tsai,An Efficient and Accurate Camera Calibration Technology for 3D Machine Vision. Proceeding of CVPR, IEEE. 1987:364-37 